\title{
A Putative MAPK Kinase Kinase Gene Ssos 4 is Involved in Mycelial Growth, Virulence, Osmotic Adaptation, and Sensitivity to Fludioxonil and is Essential for SsHog1 Phosphorylation in Sclerotinia sclerotiorum
}

\author{
Tao Li, ${ }^{1}$ Qian Xiu, ${ }^{1}$ Jianxin Wang, ${ }^{1,2}$ Yabing Duan, ${ }^{1,2, \dagger}$ and Mingguo Zhou ${ }^{1,2, \dagger}$ \\ ${ }^{1}$ College of Plant Protection, Nanjing Agricultural University, Nanjing 210095, China \\ 2 The Key Laboratory of Plant Immunity, Nanjing Agricultural University, Nanjing 210095, China \\ Accepted for publication 5 October 2020
}

\begin{abstract}
The high osmolarity glycerol (HOG) pathway, comprising a twocomponent system and the $\operatorname{Hog} 1$ mitogen-activated protein kinase (MAPK) cascade, plays a pivotal role in eukaryotic organisms. Previous studies suggested that the biological functions of some key genes in the HOG pathway varied in filamentous fungi. In this study, we characterized a putative MAPK kinase kinase gene, Ssos4, in Sclerotinia sclerotiorum, which encoded a phosphotransferase in the MAPK cascade. Compared with the wild-type progenitor HA61, the deletion mutant $\Delta$ Ssos4-63 exhibited impaired mycelial growth, sclerotia formation, increased hyphal branches, and decreased virulence. The deficiencies of the deletion mutant $\Delta \mathrm{S}$ sos4-63 were recovered when the full-length Ssos 4 gene was complemented. Deletion of Ssos 4 increased the sensitivity to osmotic stresses and cell wall agents and the resistance to fludioxonil and dimethachlon. Intracellular glycerol accumulation was not induced in the deletion mutant $\Delta S \operatorname{Ssos} 4-63$ when treated with
\end{abstract}

ABSTRACT fludioxonil and $\mathrm{NaCl}$ and the phosphorylation of $\mathrm{SsHog} 1$ was also cancelled by the deletion of Ssos4. Consistent with the glycerol accumulation and increased expression levels of $S \operatorname{sg} l p A$ and $S s f p s l$, controlling glycerol synthesis and close of glycerol channel under hyperosmotic stress, respectively, were detected in the wild-type strain HA61 but not in the deletion mutant $\Delta$ Ssos4-63. Moreover, the relative expression level of Sshogl significantly decreased, whereas the expression level of $S \operatorname{sos} 5$ increased in the deletion mutant $\Delta$ Ssos463. These results indicated that Ssos 4 played important roles in mycelial growth and differentiation, sclerotia formation, virulence, hyperosmotic adaptation, fungicide sensitivity, and the phosphorylation of SsHog1 in S. sclerotiorum.

Keywords: fungicide sensitivity, MAPK cascade, fludioxonil, mycelial growth, osmotic sensitivity, phosphorylation, Sclerotinia sclerotiorum
Sclerotinia sclerotiorum is a cosmopolitan, necrotrophic, and devastating phytopathogen with an extensive host range (Boland and Hall 1994). During flower blossom, ascospores are deposited on the flowers and cause disease development (Johnson and Atallah 2014), and S. sclerotiorum maintains long-term survival with the pigmented, multihyphal structure of sclerotia (Bolton et al. 2006). In China, Sclerotinia stem rot (SSR) caused by S. sclerotiorum is the most important disease of oilseed rape, leading to serious economic losses in the field. Due to the lack of resistant cultivars, the application of chemical fungicides is a primary measure for controlling SSR. Many chemical fungicides, including tebuconazole, boscalid, fluazinam, and trifloxystrobin, could effectively inhibit mycelial growth of S. sclerotiorum (Dalili et al. 2015; Di et al. 2016; Liu et al. 2018, 2019; Wang et al. 2009). However, with extensive and longterm use in China, resistance to some fungicides has occurred in the field (Ma et al. 2009; Pan et al. 1997; Zhu et al. 2016). Fludioxonil is a phenylpyrrole fungicide and that exhibits excellent activity against $S$. sclerotiorum (Duan et al. 2013a) but its action mechanism remains unclear.

†Corresponding authors: Y. Duan; dyb@njau.edu.cn; and M. Zhou; mgzhou@njau.edu.cn

Funding: Support was provided by The National Natural Science Foundation of China (grant number 31401764), Jiangsu Agriculture Science and Technology Innovation Fund (JASTIF) (CX(19)3110), and The Fundamental Research Funds for the Central Universities (grant number KJQN201508).

*The $\boldsymbol{e}$-Xtra logo stands for "electronic extra" and indicates that a supplementary table and supplementary figures are published online.

The author(s) declare no conflict of interest.

(c) 2021 The American Phytopathological Society
The high osmolarity glycerol (HOG) pathway is an important system in adaptation to hyperosmotic and oxidative stresses in prokaryotes and eukaryotes. It consists of a two-component signal system and the Hog 1 mitogen-activated protein kinase (MAPK) cascade (Posas et al. 1996). The osmosensors in the two-component system sense extracellular hyperosmotic stresses and regulate the accumulation of several organic molecules to balance the osmotic pressure in cells with that of the external environment through the activation of MAPK cascades (de Nadal et al. 2002). A variety of cellular processes, including cell fate and innate immunity in mammalian cells and plant defense, are modulated by MAPK cascades (Arthur and Ley 2013; Meng and Zhang 2013; Zhang and Klessig 2001). In filamentous fungi, the HOG pathway also plays various vital functions. Osmosensor histidine kinases (HKs) mediate mycelial differentiation, sclerotia formation, virulence, response to stresses, and fungicide sensitivity in fungi (Avenot et al. 2005; Duan et al. 2013b; Jacob et al. 2014; Viaud et al. 2006). In Neurospora crassa, Ncrrg-1,Ncos-4, and Ncos-2 genes are involved in responses to osmotic stress and sensitivity to iprodione and fludioxonil (Fujimura et al. 2003; Jones et al. 2007; Noguchi et al. 2007). The phosphorylation of Hog1, which is located downstream of the HOG pathway, is regulated by HK and is involved in stress response, microsclerotia formation, hyphal growth, and plant infection (Wang et al. 2016; Zheng et al. 2012). Considering its important roles in organisms, the HOG pathway was thought to be a potential antifungal drug target (Jacob et al. 2015; Shor and Chauhan 2015).

Under hyperosmotic stress, the osmosensor $\operatorname{Sln} 1$ is inactive, renders Ssk1 dephosphorylated, and activates the Pbs2-Hog1 module by activating the redundant MAPK kinase kinases (MAPKKKs) Ssk2p and Ssk22p in yeast (Maeda et al. 1994; Posas et al. 1996). However, HOG activation is dependent on the MAPKKK Ssk2 rather than its paralog Ssk22 in the Sln1 branch (Jiang et al. 2014). Due to its specific role in the HOG pathway, 
the functions of Ssk2 were uncovered in various fungi. In Saccharomyces cerevisiae, Ssk2p is specialized to facilitate actin cytoskeleton reassembly after osmotic stress (Yuzyuk et al. 2002). In $\mathrm{N}$. crassa, the $\mathrm{NcOs}$-4 deletion mutant is sensitive to osmotic stress and resistant to iprodione and fludioxonil (Fujimura et al. 2003). In Cryptococcus neoformans, Ssk2 not only regulates hyperosmotic adaptation and fludioxonil sensitivity but also is involved in the regulation of capsule and melanin biosynthesis (Bahn et al. 2007). Fgos4 regulates pigmentation and production of trichothecenes in the aerial hyphae of Fusarium graminearum and $B \cos 4$ is involved in vegetative differentiation, virulence, fungicide responses, and adaptation to hyperosmotic and xidative stresses in Botrytis cinerea (Ochiai et al. 2007; Yang et al. 2012). However, the function of $S s k 2$ is still unclear in $S$. sclerotiorum. In this study, we cloned the homolog paralog of $S s k 2$ and studied its characterization in S. sclerotiorum.

\section{MATERIALS AND METHODS}

Fungal strain and culture conditions. S. sclerotiorum wildtype strain HA61 was used as a progenitor for the fungal protoplast transformation experiment. The strain was isolated from rapeseed in Jiangsu Province of China and is sensitive to phenylpyrrole and dicarboximide fungicides (Duan et al. 2013b). Potato dextrose agar (PDA) medium was used to incubate strains and test their sensitivity to fungicides and other stresses. Yeast-peptone-glucose (YEPD) medium ( $10 \mathrm{~g}$ of peptone, $20 \mathrm{~g}$ of glucose, and $1 \mathrm{~g}$ of yeast extract per liter) was used for mycelium cultivation.

Construction of the Ssos 4 null allele and protoplast transformation. The knockout construct was generated by fusion PCR with three DNA fragments. First, a 1,310-bp upstream fragment and a 1,267-bp downstream fragment of Ssos 4 were amplified from genomic DNA (gDNA) of HA61 with the primer pairs P1/P2 and
$\mathrm{P} 3 / \mathrm{P} 4$, respectively. The 1,764-bp fragment of hygromycin phosphotransferase $(\mathrm{hph})$ gene under control of the Aspergillus nidulans trpC Promoter (PtrpC) was amplified from pKht (Mullins et al. 2001) with the primer pair $\mathrm{P} 5 / \mathrm{P} 6$. The $5^{\prime}$ region of $\mathrm{P} 2$ and $3^{\prime}$ region of $\mathrm{P} 3$ contained a 20- to 25-bp complementary sequence of the $h p h$ cassette (Fig. 1A). Then, these two flanking fragments were fused with the $h p h$ cassette at a mole ratio of 1:3:1 (upstream/hph cassette/ downstream) and the fusion fragment was used as a template to amplify the $S$ sos 4 null allele with primer pair P1/P4 in a $5 \times$ diluted concentration.

The 4,341-bp fragment was used for protoplast transformation according to the previous method, with minor modifications (Ge et al. 2013; Liu et al. 2013). When hygromycin-resistant colonies appeared, the single mycelial tips of the colonies were transferred to PDA amended with hygromycin B at $100 \mu \mathrm{g} / \mathrm{ml}$ three times. Then, the gDNA of the transformants was extracted by the cetyltrimethylammonium bromide method, with minor improvements, and the correct transformants with homologous insertion were detected by the primer pairs P7/P8, P9/P10, and P11/P12 (Fig. 1B, C, and D). All of the primers used in this study are listed in Supplementary Table S1.

Gene complementation. To confirm that the phenotype of the deletion mutant $\Delta$ Ssos4-63 was caused by the deletion of $S \operatorname{sos} 4$, the full-length Ssos 4 gene was complemented to the deletion mutant $\Delta$ Ssos4-63. For the complementary experiment, a modified plasmid pNEO (Duan et al. 2013b) was used. The full-length Ssos 4 gene, containing a 1,470-bp upstream flanking sequence and a 816-bp downstream flanking sequence, was amplified from gDNA of the wild-type progenitor HA61 with primer pair P13/P14 (Supplementary Table S1). Then, the 6,314-bp fragment was cloned to the XmaI site of pNEO to generate the complementary plasmid pNEO-Ssos4Com with ClonExpress II One Step Cloning Kit (Vazyme Biotech Co. Ltd.). The recombinant plasmid for complementation was
A
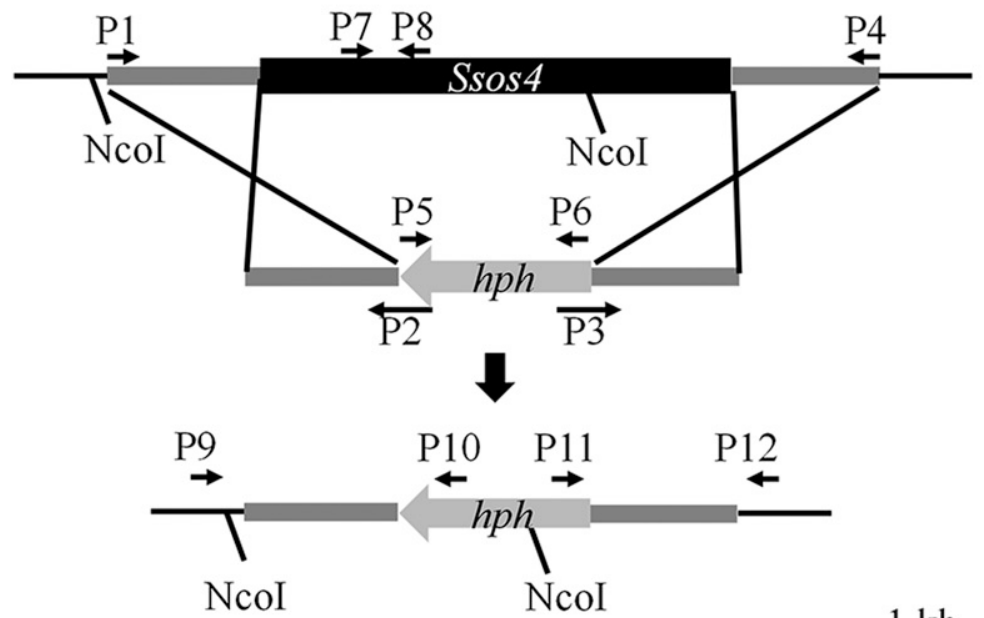

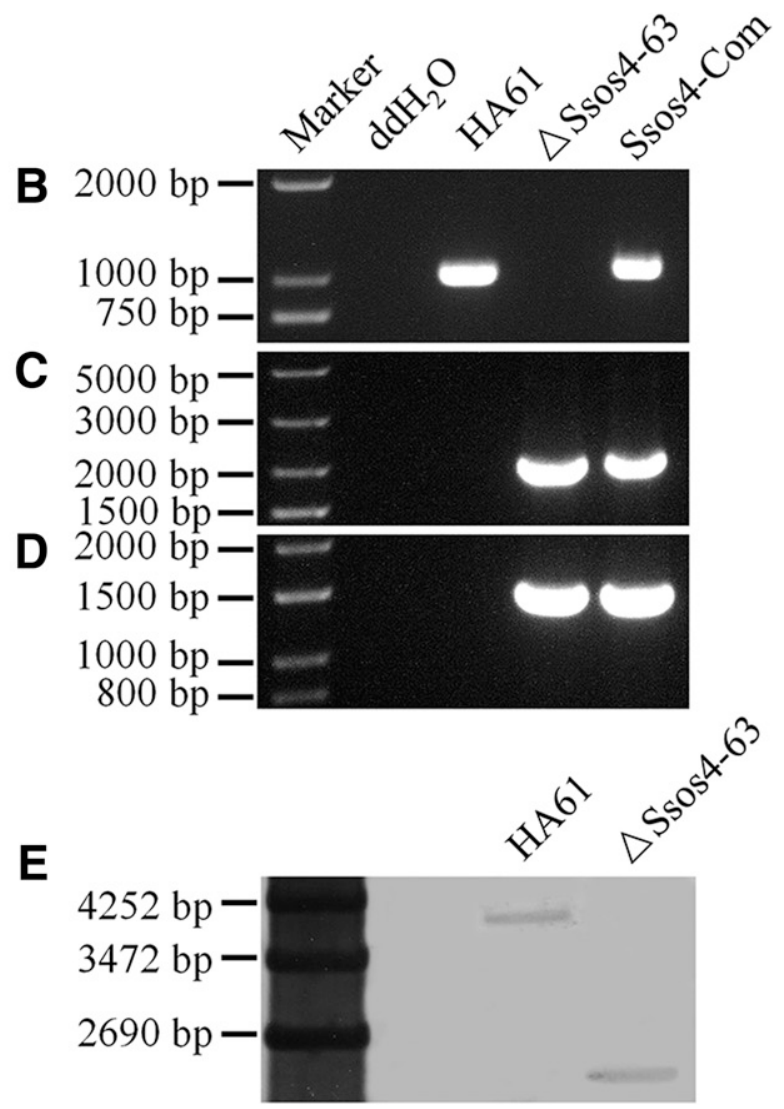

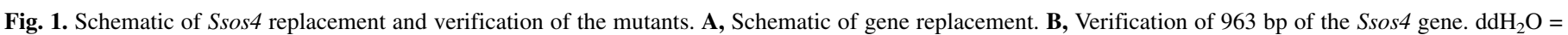

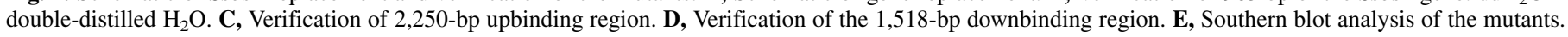


cloned and sequenced before transformation. Transformation of the deletion mutant $\Delta$ Ssos4-63 with the full-length $S \operatorname{sos} 4$ was performed as described above, except that neomycin $(100 \mu \mathrm{g} / \mathrm{ml})$ was used as the selected agent.

Southern blot analysis. To further identify the null mutation, Southern blot analysis was performed. The probe of the 672-bp fragment was amplified by the primer pair P15/P16 (Supplementary Table S1) and labeled by digoxin. The gDNA of the wild-type progenitor HA61 and the deletion mutant $\triangle \mathrm{Ssos} 4-63$ were digested by $\mathrm{NcoI}$ and the digested fragments were separated by agarose gel electrophoresis (Fig. 1E). The subsequent processes were performed as described in the manufacturer's protocol (F. Hoffmann-La Roche Ltd.).

Mycelia growth, sclerotia production, and mycelial morphology. To characterize the phenotypic change, the wildtype progenitor HA61 and the deletion mutant $\Delta$ Ssos4-63 were assayed on PDA plates. For mycelia growth, the strains were incubated on PDA plates for 2 days at $25^{\circ} \mathrm{C}$ and, for the sclerotia formation test, the strains were incubated for 15 days. Moreover, hyphae morphology was also observed by optical microscope (Zeiss Primo Star) after incubation on PDA plates for $24 \mathrm{~h}$.

Virulence test. The virulence of the wild-type progenitor HA61 and the deletion mutant $\Delta$ Ssos4-63 were tested on healthy rapeseed leaves. Mycelia plugs with a 5-mm diameter from the edge of 2-dayold colonies were used to inoculate the leaves. Before inoculation, the leaves were rinsed and wounded by a sterilized pin to facilitate infection. After incubation for 2 days in a chamber with $85 \%$ humidity and $16 \mathrm{~h}$ of daylight at $25^{\circ} \mathrm{C}$, the diameters of lesions were measured. The experiment was performed two times.

Oxalic acid production. Oxalic acid was considered to be a pathogenic factor in S. sclerotiorum (Guimarães and Stotz 2004; Williams et al. 2011). To assay the effect of Ssos 4 deletion on oxalic acid production, oxalic acid secreted by the wild-type progenitor HA61 and the deletion mutant $\Delta$ Ssos4-63 was tested. Ten mycelial plugs from the edge of 2-day-old colonies were transferred into $100 \mathrm{ml}$ of YEPD and incubated in a shaker at $175 \mathrm{rpm}$ and $25^{\circ} \mathrm{C}$. After incubation for $36 \mathrm{~h}$, the mycelia were harvested with microcloth and the filtrate was collected for the oxalic acid test. To determine the concentration of oxalic acid, $2 \mathrm{ml}$ of $\mathrm{FeCl}_{3}$ $(0.5 \mathrm{mg} / \mathrm{ml}), 20 \mathrm{ml}$ of $\mathrm{HCl}-\mathrm{KCl}$ buffer $(50 \mathrm{mM} \mathrm{KCl}, \mathrm{PH} 2.0), 1.2 \mathrm{ml}$ of sulfosalicylic acid $(5 \mathrm{mg} / \mathrm{ml})$, and $1 \mathrm{ml}$ of filtrate were added sequentially to a 50-ml centrifuge and the mixture was filled to $25 \mathrm{ml}$ with double-distilled $\mathrm{H}_{2} \mathrm{O}$. Subsequently, the mixture was vortexed for $10 \mathrm{~s}$ and incubated for $30 \mathrm{~min}$ at $25^{\circ} \mathrm{C}$, and the optical density at $510 \mathrm{~nm}$ was measured using a Versa Max Pro 5 microplate reader (Molecular Device Inc.). The concentration of oxalic acid was computed according to the standard curve. Each treatment had three replicates and the experiment was repeated twice.

Stress responses and fungicide sensitivity. To test the responses of the strains to high osmotic stress, the wild-type progenitor HA61 and the deletion mutant $\Delta$ Ssos4-63 were incubated on PDA plates containing $0.4 \mathrm{M} \mathrm{NaCl}, 0.4 \mathrm{M} \mathrm{KCl}, 1 \mathrm{M}$ glucose, or $0.8 \mathrm{M}$ sorbitol. For membrane and cell wall stress tests, the strains were incubated on PDA plates with $0.01 \%$ sodium dodecyl sulfate (SDS) and Congo red at $0.2 \mathrm{~g} /$ liter. After incubation for 2 days at $25^{\circ} \mathrm{C}$, the diameters of the colonies were measured and the relative growth rate was calculated. Moreover, to test the sensitivity change of $S \operatorname{sos} 4$ deletion, the wild-type progenitor HA61 and the deletion mutant $\Delta$ Ssos4-63 were incubated on PDA amended with fludioxonil and dimethachlon. For tests of the half maximal effective concentration $\left(\mathrm{EC}_{50}\right)$, the concentrations of fludioxonil were $0,0.00125,0.0025,0.005,0.01,0.02$, and $0.04 \mu \mathrm{g} / \mathrm{ml}$ and the concentrations of dimethachlon were $0,0.125,0.25,0.5,1$, and $2 \mu \mathrm{g} / \mathrm{ml}$. To test the resistance change, much higher doses of fungicides were used. The concentrations were $0.1,1$, and $10 \mu \mathrm{g} / \mathrm{ml}$ for fludioxonil and 1,5 , and $10 \mu \mathrm{g} / \mathrm{ml}$ for dimethachlon.

Glycerol accumulation. To identify the effect of Ssos 4 deletion on glycerol accumulation, glycerol contents in mycelia of the wild-type strain HA61 and the deletion mutant $\Delta$ Ssos4-63 were tested. For the glycerol content assay, 10 mycelia plugs from the edge of 2-day-old colonies were transferred into $100 \mathrm{ml}$ of YEPD and incubated with shaking at $175 \mathrm{rpm}$ and $25^{\circ} \mathrm{C}$. After incubation for $36 \mathrm{~h}$, fludioxonil at $0.1 \mu \mathrm{g} / \mathrm{ml}$ and $0.4 \mathrm{M} \mathrm{NaCl}$ were added to the cultures and incubated for an additional $1 \mathrm{~h}$. The mycelia were harvested with microcloth and air dried. The extraction and measurement processes were performed as described in the manufacturer's protocols (Applygen Technologies Inc.). The glycerol concentration was calculated with a standard curve and the experiments were repeated twice.

Western blot analysis. To analyze the effect of Ssos 4 deletion on the phosphorylation of $\mathrm{SsHog} 1$, five plugs from the edge of 2-day-old colonies were transferred into $100 \mathrm{ml}$ of YEPD and incubated in a shaker at $175 \mathrm{rpm}$ and $25^{\circ} \mathrm{C}$. After incubation for 36 $\mathrm{h}$, fludioxonil at $0.1 \mu \mathrm{g} / \mathrm{ml}$ and $0.4 \mathrm{M} \mathrm{NaCl}$ were added to the cultures and incubated for an additional $1 \mathrm{~h}$. The mycelia were harvested and the total protein of the wild-type strain HA61 and the deletion mutant $\Delta$ Ssos4- 63 were extracted by the method described by Yang et al. (2012) and the extract was stored at $-80^{\circ} \mathrm{C}$ for use. To detect phosphorylated SsHog1, the antip38 MAPK (PhosphoThr180/Tyr182) rabbit pAb (catalog number 382888; Zen BioScience) was used. A p38 mouse mAb (catalog number 200782; Zen BioScience) was used to detect the total SsHog1 and the experiment was performed twice.

RNA extraction and quantitative PCR analysis. The total RNA of the wild-type progenitor HA61 and the deletion mutant $\triangle$ Ssos463 was extracted using an RNA Extraction Kit (Tiangen Biotech Co. Ltd.). For mycelia preparation, five mycelial plugs from the edge of a 2-day-old colony were transferred into $100 \mathrm{ml}$ of YEPD and incubated in a shaker at $175 \mathrm{rpm}$ and $25^{\circ} \mathrm{C}$. After incubation for $36 \mathrm{~h}$, mycelia were harvested and used for RNA extraction. RNA concentration was determined and reverse transcription was conducted with HiScript II Q RT SuperMix (Vazyme Biotech Co. Ltd.). According to the protocols described, quantitative PCR (qPCR) was performed with ChamQ SYBR qPCR Master Mix (Vazyme Biotech Co. Ltd.). The amplification of genes in the HOG pathway was conducted with primer pairs P17/P18, P19/P20, P21/P22, P23/P24, P25/P26, and P27/P28 (Supplementary Table S1). Ssgapdh (SS1G_07798) was selected as an internal reference and was amplified by P29/P30. The relative expression levels were calculated with the $2^{-\Delta \mathrm{Ct}}$ method and the experiments were repeated twice.

\section{RESULTS}

Identification and characterization of Ssos4. The Ssos4 gene was identified by blasting ssk2 of Saccharomyces cerevisiae in the database of the Kyoto Encyclopedia of Genes and Genomes and the orthology gene SS1G_06598 was found in S. sclerotiorum. Then, we blasted the amino acid sequence of Ssos 4 with that of the model fungi and several plant pathogens, and a neighbor-joining tree was generated using MEGA7 by the bootstrap method (Fig. $2 \mathrm{~A})$. The result showed that $S$. sclerotiorum was classified in the same branch with $B$. cinerea. The complementary DNA (cDNA) and gDNA of Ssos 4 from the wild-type strain HA61 were amplified, sequenced. and blasted with the sequence from NCBI (GenBank ID: XM_001592307.1). The results showed that the full length of the Ssos 4 gene was 4,028 bp and the cDNA length was 3,972 nucleotides encoding a protein of 1,324 amino acids. To further reveal the protein structure, the amino acid sequence was predicted with the SMART prediction server (http://smart.embl-heidelberg.de/) and a serine/threonine protein kinase catalytic domain was detected which belongs to a serine- or threonine-specific kinase subfamily functioning as a phosphotransferase (Fig. 2B). All of these results indicated that $S \operatorname{sos} 4$ encoded a MAPKKK and functioned as a protein serine/threonine kinase transferring phosphoruscontaining groups.

Deletion and complementation of Ssos4. To elucidate the biological function of Ssos 4 in S. sclerotiorum, an Ssos 4 null allele was generated by replacing $S \operatorname{sos} 4$ with a hygromycin resistance 
cassette using the fusion PCR method (Fig. 1A). By polyethyleneglycol-mediated protoplast transformation and selection on PDA plates three times with hygromycin B at $100 \mu \mathrm{g} / \mathrm{ml}$, the mutants were screened. Furthermore, PCR and Southern blot methods were conducted to verify the $S s o s 4$ deletion mutants. In PCR verification, a fragment of $963 \mathrm{bp}$ of the Ssos 4 gene was amplified from the wild-type progenitor HA61 and the complementary strain rather than the deletion mutant $\Delta$ Ssos4-63 (Fig. 1B). Fragments of the 2,250-bp upbinding region and 1,518-bp downbinding region were amplified from the deletion mutant $\Delta$ Ssos4-63 as well as in the complementary strain rather than the wild-type progenitor HA61 (Fig. 1C and D). The results indicated that the null Ssos4 allele had replaced the $S \operatorname{sos} 4$ locus after homologous recombination. In Southern blot analysis, a 4,040-bp band was detected in the wild-type strain HA61 whereas it was 2,373 bp in the deletion mutant $\Delta$ Ssos4-63 (Fig. 1E), suggesting that the null Ssos 4 allele had replaced the $S$ sos 4 allele as a single copy in the deletion mutant $\Delta$ Ssos4-63.

Ssos4 involved in mycelial growth, sclerotia formation, and hyphal differentiation in $S$. sclerotiorum. It has been reported that MAPKKK was involved in the regulation of mycelial growth (Yang et al. 2012). Here, mycelial growth of the deletion mutant $\Delta$ Ssos4-63 was measured at an interval of $12 \mathrm{~h}$ on PDA plates. Compared with the wild-type progenitor HA61, the growth rate of the deletion mutant $\Delta$ Ssos4-63 decreased significantly (Fig. 3A and B). After incubation for 2 days, the colony diameter of the wild-type progenitor HA61 was $8.66 \pm 0.01 \mathrm{~cm}$ whereas it was only $3.60 \pm$ $0.03 \mathrm{~cm}$ for the deletion mutant $\Delta \mathrm{S}$ sos4-63. Sclerotia formation was also investigated on PDA plates. The results showed that the deletion mutant $\Delta$ Ssos4-63 exhibited a significant decrease in sclerotia production in comparison with the wild-type progenitor HA61 (Fig. $3 \mathrm{C}$ and $\mathrm{D})$. In addition, the deletion mutant $\Delta$ Ssos4-63 grew denser and showed more branches than the wild-type progenitor HA61 (Fig. $3 \mathrm{E})$. All of the defects in the deletion mutant were recovered by the complementary strain of Ssos4. These results indicated that Ssos 4 had pleiotropic effects on mycelial growth, sclerotia formation, and hyphal differentiation in S. sclerotiorum.
Ssos 4 is involved in the virulence of $S$. sclerotiorum. The deletion mutant $\Delta$ Ssos4-63 and the wild-type progenitor HA61 were inoculated on detached rapeseed leaves that were wounded with pins or not wounded. Compared with the wild-type progenitor HA61, the deletion mutant $\Delta \mathrm{Ssos} 4-63$ showed significant impaired virulence (Fig. 4A). On the wounded leaves, the average size of lesions was $0.18 \pm 0.04 \mathrm{~cm}^{2}$ when inoculated with the deletion mutant $\Delta$ Ssos4-63, whereas it was $4.88 \pm 0.99 \mathrm{~cm}^{2}$ for the wild-type progenitor HA61 (Fig. 4C). However, the deletion mutant $\Delta$ Ssos4-63 was hardly pathogenic on the intact leaves (Fig. $4 \mathrm{~A}$ and $\mathrm{C}$ ). The virulence was rescued by the complementary strain of Ssos 4 on either the wounded or intact leaves. To further explore the reason for the decreased virulence of the deletion mutant $\Delta$ Ssos4-63 and formation of the infection cushion, the infection structure of $S$. sclerotiorum was analyzed. The results showed that infection cushions formed in the deletion mutant $\triangle$ Ssos4-63 were smaller and fewer than in the wild-type strain HA61, suggesting that deletion of Ssos 4 decreased formation of infection cushion (Fig. 4B). According to previous reports, oxalic acid was considered to be an important virulence factor in S. sclerotiorum (Godoy et al. 1990; Jamaux et al. 1995). Therefore, the production of oxalic acid was tested. The results showed that there was no difference in oxalic acid production between the wild-type progenitor HA61 and the deletion mutant $\Delta$ Ssos4-63 (Fig. 4D). Taken together, the results showed that Ssos 4 was involved in the formation of the infection cushion to facilitate infection progress and pathogenicity in S. sclerotiorum.

Deletion of Ssos4 results in defects in hyperosmotic responses. MAPKKK is an important phosphate-transmitting protein and plays an important role in adaptation to hyperosmotic stress. To understand the function of Ssos 4 in osmotic adaptation, we assayed the growth of the $S \operatorname{sos} 4$ deletion mutant on PDA in the presence of osmotic agents. The deletion mutant $\Delta$ Ssos4-63 showed significant defects of mycelial growth in the presence of $0.4 \mathrm{M}$ $\mathrm{NaCl}, 0.4 \mathrm{M} \mathrm{KCl}$, and $0.8 \mathrm{M}$ sorbitol (Fig. 5A and B). Under the same conditions, the wild-type progenitor HA61 was barely affected by osmotic stresses. Surprisingly, in the presence of $1 \mathrm{M}$

A

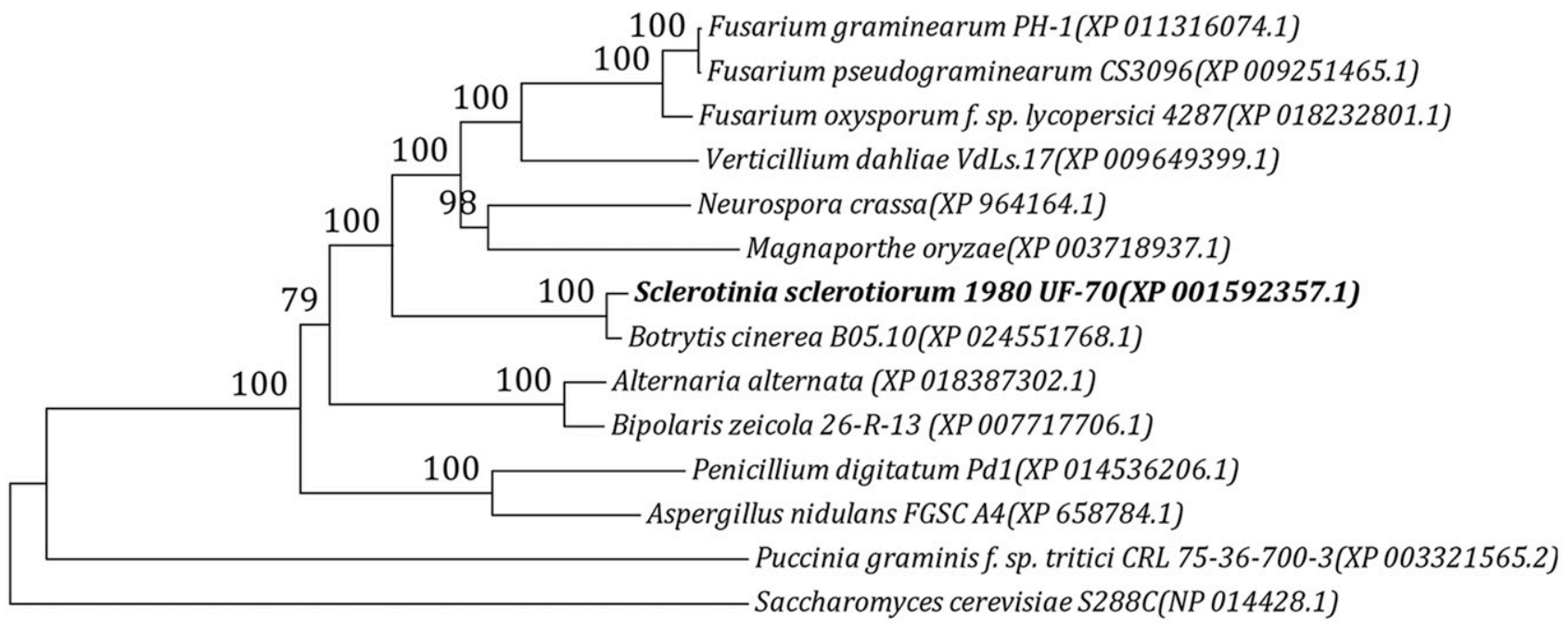

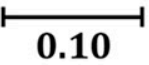

B

LCR LCR

CCR S_TKe 1022-1293 aa LCR

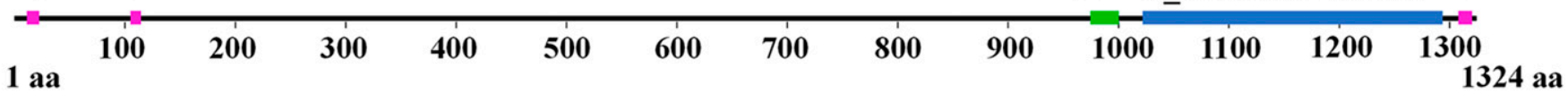

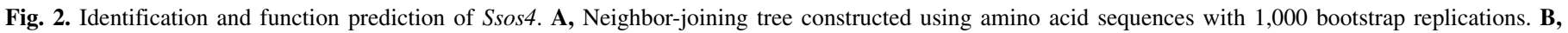
SMART prediction of Ssos4 protein. 
glucose, mycelial growth was inhibited for either the wild-type progenitor HA61 or the deletion mutant $\triangle$ Ssos4-63.

Ssos 4 is involved in responses to cell wall and membrane stresses. We also tested the responses of the deletion mutant $\Delta$ Ssos4-63 to cell wall and membrane stresses. In the presence of Congo red at $0.2 \mathrm{~g} / \mathrm{liter}$ as cell wall stress, the deletion mutant $\Delta$ Ssos4-63 was significantly inhibited in comparison with the wild-type strain HA61. However, after incubation on PDA with $0.01 \%$ SDS added, the deletion mutant $\triangle$ Ssos4-63 was more tolerant than the wild-type strain HA61 (Fig. 5A and B). The alterations of the responses to stresses were rescued by complementing Ssos 4 to the deletion mutant $\Delta$ Ssos4-63. The above results suggested that $\operatorname{Ssos} 4$ positively regulates the response to cell wall stress and negatively regulates the response to cell membrane stress.

Ssos 4 is involved in fungicide sensitivity. To investigate whether $S \operatorname{sos} 4$ is involved in the regulation of fungicide sensitivity, we assayed the sensitivities of the mutants to fludioxonil and dimethachlon. The $\mathrm{EC}_{50}$ value of fludioxonil was $0.0046 \pm$ $0.0004 \mu \mathrm{g} / \mathrm{ml}$ for the wild-type progenitor HA61 and it was significantly increased to $0.0080 \pm 0.0003 \mu \mathrm{g} / \mathrm{ml}$ for the deletion mutant $\Delta \operatorname{Ssos} 4-63(P=0.0032)$. For dimethachlon, the $\mathrm{EC}_{50}$ value was $0.6364 \pm 0.0450 \mu \mathrm{g} / \mathrm{ml}$ for the wild-type progenitor HA61 and it was reduced to $0.4838 \pm 0.0125 \mu \mathrm{g} / \mathrm{ml}$ for the deletion mutant $\triangle$ Ssos4-63 $(P=0.0252)$. Nevertheless, the wild-type progenitor HA61 was $100 \%$ inhibited on PDA with much higher concentrations of fungicides whereas the deletion mutant $\Delta$ Ssos4-63 survived, although the growth was severely inhibited (Fig. 6). The sensitivity was recovered by the complementary strain of Ssos 4 . This suggested that $\operatorname{Ssos} 4$ was involved in the regulation of sensitivity to fludioxonil and dimethachlon in S. sclerotiorum.

Ssos 4 regulates glycerol accumulation and the phosphorylation of SsHog1. It has been reported that glycerol accumulation was induced in response to hyperosmotic stress and fludioxonil in N. crassa and Saccharomyces cerevisiae via activation of the HOG pathway (Lew 2010; Wojda et al. 2003). Therefore, we analyzed glycerol accumulation in the mycelia after exposure to fludioxonil at $0.1 \mu \mathrm{g} / \mathrm{ml}$ and $0.4 \mathrm{M} \mathrm{NaCl}$ for $1 \mathrm{~h}$. The results showed that fludioxonil and $\mathrm{NaCl}$ treatments significantly
A HA61
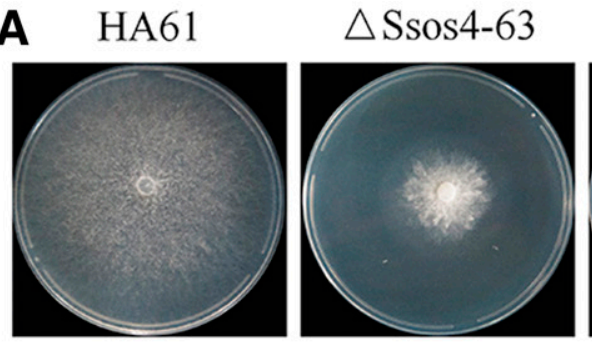

B

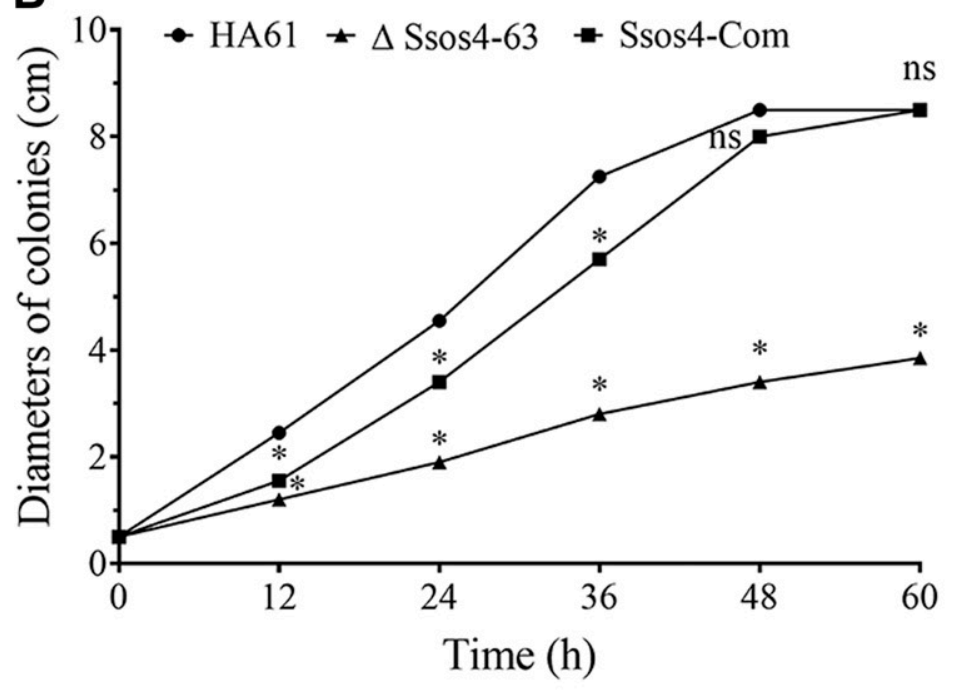

$\mathbf{E}$

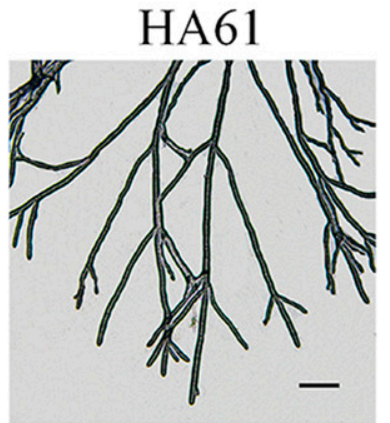

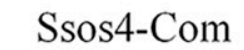

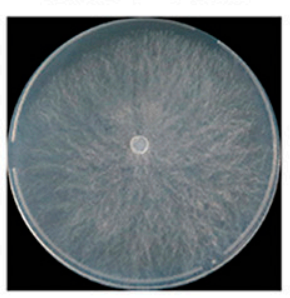

C HA61

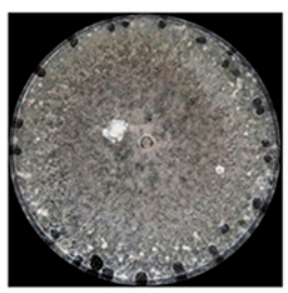

D

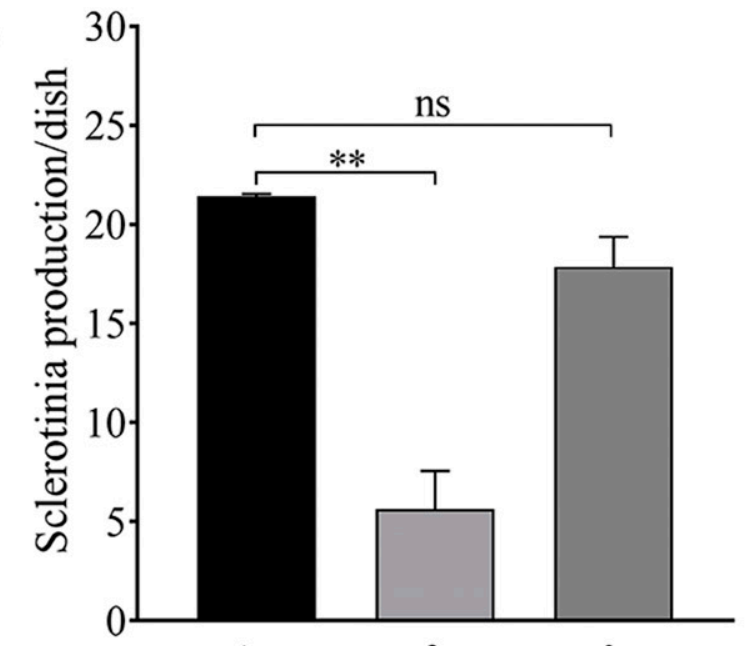

$\triangle \operatorname{Ssos} 4-63$

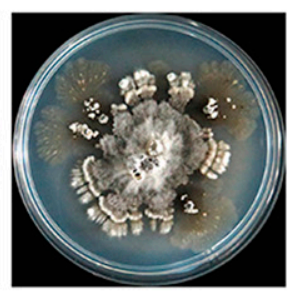

Ssos4-Com

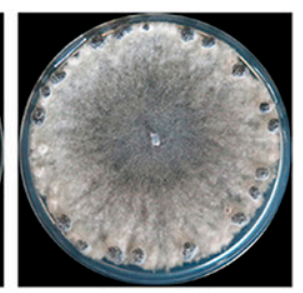

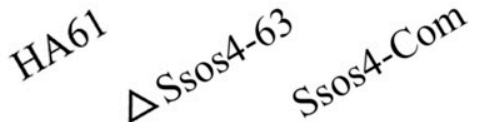
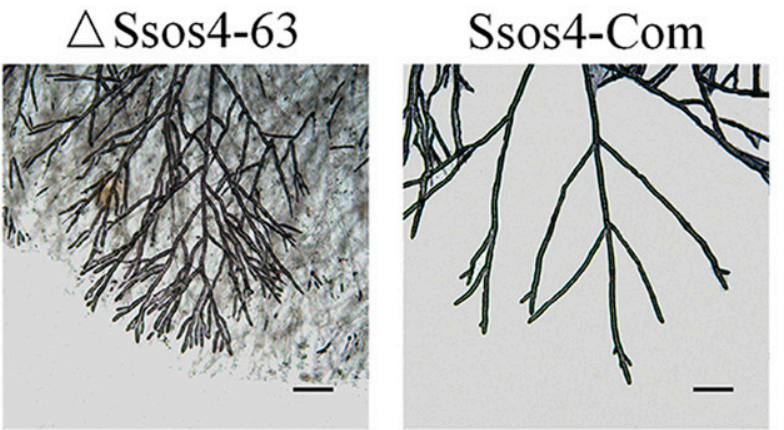

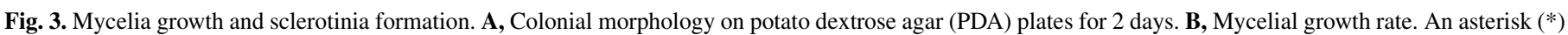

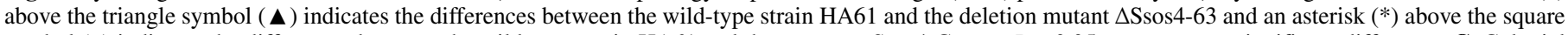

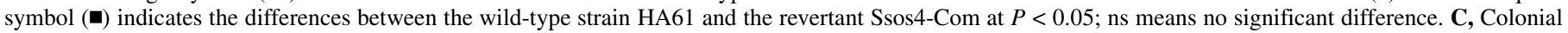

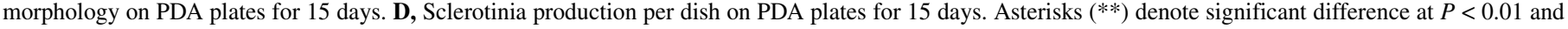
ns denotes no significant difference at $P<0.05$. E, Hyphal morphology on PDA plates observed with optical microscope. Black bar $=200 \mu \mathrm{m}$. 
increased glycerol accumulation in the wild-type progenitor HA61 and the complementary strain Ssos4-Com rather than in the deletion mutant $\Delta$ Ssos4-63 (Fig. 7A). The difference in accumulation of intracellular glycerol suggested that Ssos 4 was involved in the regulation of intracellular glycerol accumulation in response to $\mathrm{NaCl}$ and fludioxonil in S. sclerotiorum.

Phosphorylation of Hog1 plays a vital role in regulating the response to osmotic stresses. In order to reveal the effect of Ssos 4 on signal transmission in the Hog1 MAPK cascade, the phosphorylation of SsHog1 was examined. The phosphorylated SsHog1 was detected in response to fludioxonil at $0.1 \mu \mathrm{g} / \mathrm{ml}$ and $0.4 \mathrm{M} \mathrm{NaCl}$ in the wild-type strain HA61. However, the phosphorylated SsHog1 was not detected in the deletion mutant $\Delta$ Ssos4-63 when treated with fludioxonil at $0.1 \mu \mathrm{g} / \mathrm{ml}$ and $0.4 \mathrm{M} \mathrm{NaCl}$ as well as in the control (Fig. 7B). In the complementary strain Ssos4-Com, the phosphorylation of SsHog1 was recovered. As a control, SsHog1 was detected in all strains. These results strongly indicated that Ssos4 was indispensable for the phosphorylation of SsHog1 in S. sclerotiorum.

Ssos4 regulates the expression of key genes in the HOG pathway. We have illustrated that the deletion of Ssos 4 affects the phosphorylation of SsHog1 and the accumulation of glycerol in response to hyperosmotic stresses and fludioxonil. To further determine the effects of Ssos 4 on the expression of key genes in the HOG pathway, quantitative reverse-transcription PCR was conducted with Ssgapdh as a reference gene. The results showed that the expression level of Sshogl decreased in the deletion mutant $\Delta$ Ssos4-63, whereas the expression level of Ssos 5 increased. In addition, the expression levels of Ssshk1, Sshpt1, and Ssssk1, the upstream genes of $S \operatorname{sos} 4$, were not affected by the deletion of $S \operatorname{sos} 4$ (Fig. 8). These results suggested that Ssos 4 gene not only regulated phosphorylation of SsHog1 but also positively regulated the expression level of Sshogl in S. sclerotiorum.

\section{DISCUSSION}

Hog1 MAPK cascades are conserved signal transmission pathways in eukaryotic cells and modulate various cellular processes such as embryogenesis, proliferation, differentiation, virulence, hyphal chemotropism, and apoptosis through signal transduction derived from the cell surface stimulated by extracellular factors (Raman et al. 2007; Wu et al. 2017). In yeast, Ssk2p is activated in an Ssk1p-dependent activation and exclusively phosphorylates the downstream Pbs2 MAPK kinase to activate the phosphorylation of Hog1 (Zhi et al. 2013). In B. cinerea, the Bcos 4 deletion mutant was remarkably impaired in vegetative growth (Yang et al. 2012) and the $\Delta \mathrm{Fgssk} 2$ mutant recorded reduced growth rate, aerial hyphal growth, and hyphal branching angle in $F$. graminearum (Zheng et al. 2012). In our study, reduced mycelial growth and increased hyphal branches were also uncovered by the disruption of Ssos 4 (Fig. 3B and E). In addition, the deletion of Ssos 4 also resulted in decreased sclerotia formation and reduced virulence (Figs. 3D and 4C), which is equivalent to reduced conidial formation and disabled infection ability in B. cinerea (Yang et al. 2012). The secretion of oxalic acid was reported to be associated with the virulence of S. sclerotiorum, and the transgenic soybean plants expressing oxalate decarboxylase
A
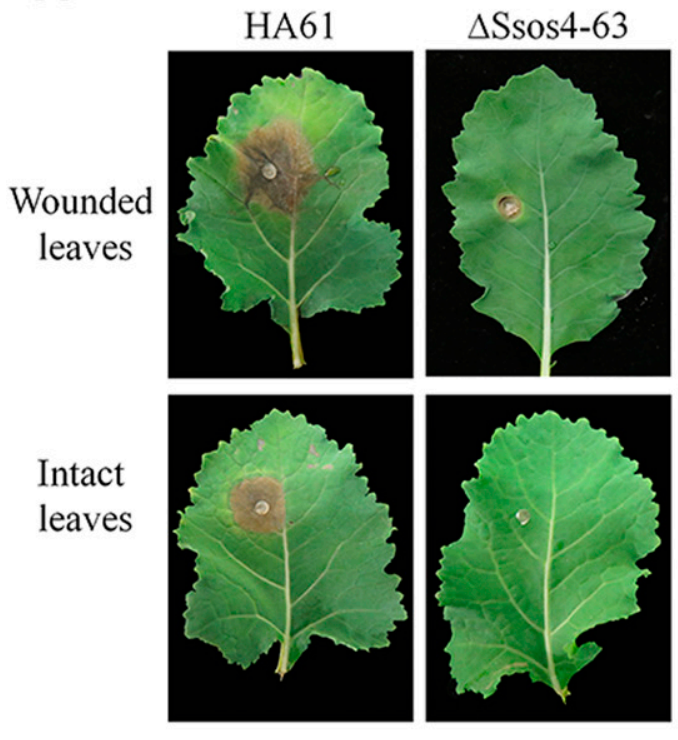

B

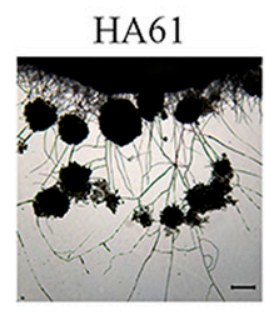

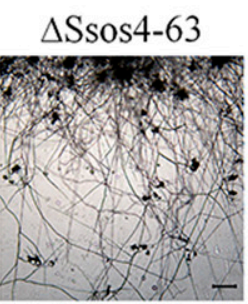

Ssos4-Com
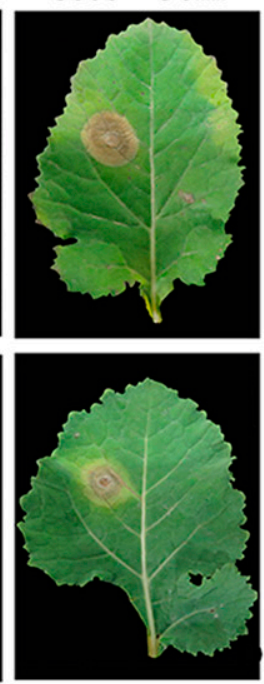

Ssos4-Com

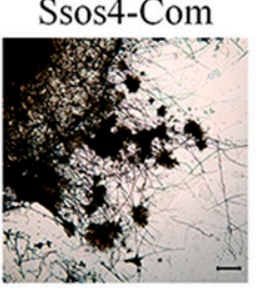

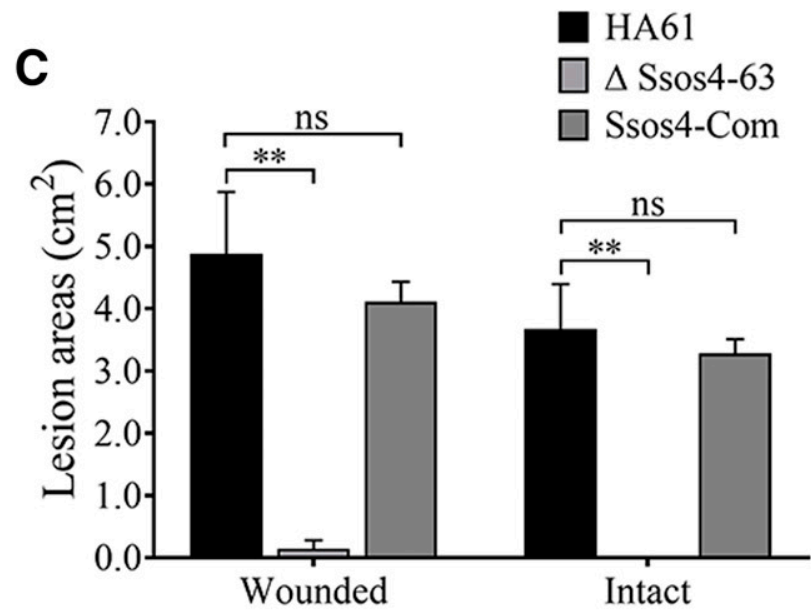

D

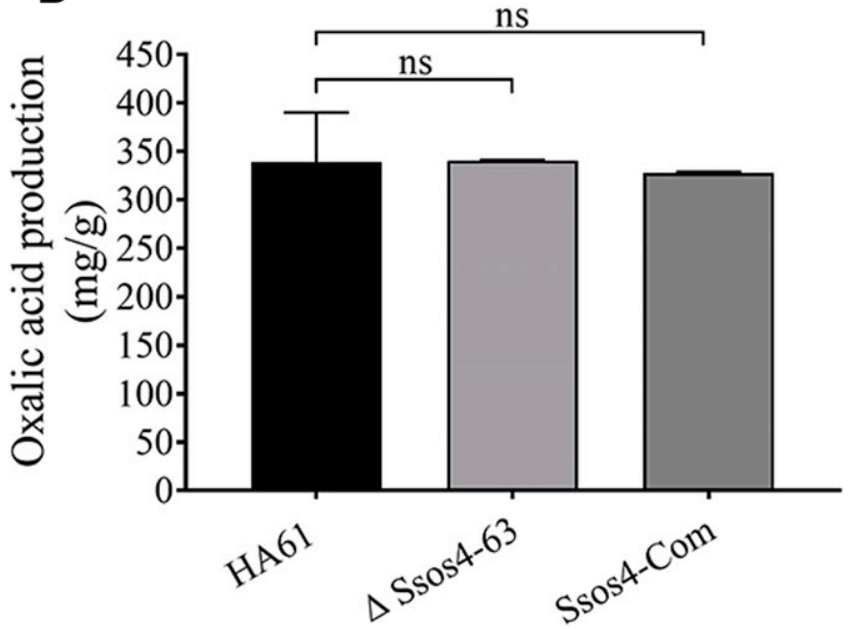

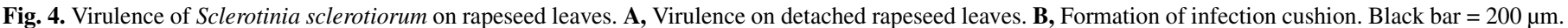

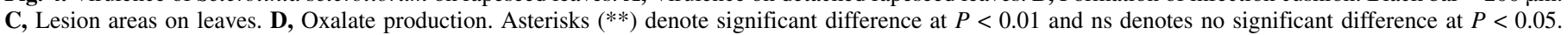


gene exhibited high resistance to $S$. sclerotiorum (Cunha et al. 2010). However, the decrease of virulence was unrelated to the oxalic acid production in the deletion mutant $\Delta$ Ssos4-63 (Fig. 4D). Moreover, decrease in the formation of infection cushions was detected in the deletion mutant $\Delta$ Ssos4-63 (Fig. 4B). Combining the results above, we speculated that the decreased virulence was due to the defect of infection cushions and the reduction of fungal growth caused by $S \operatorname{sos} 4$ deletion. To further elucidate the effect of $S \operatorname{sos} 4$

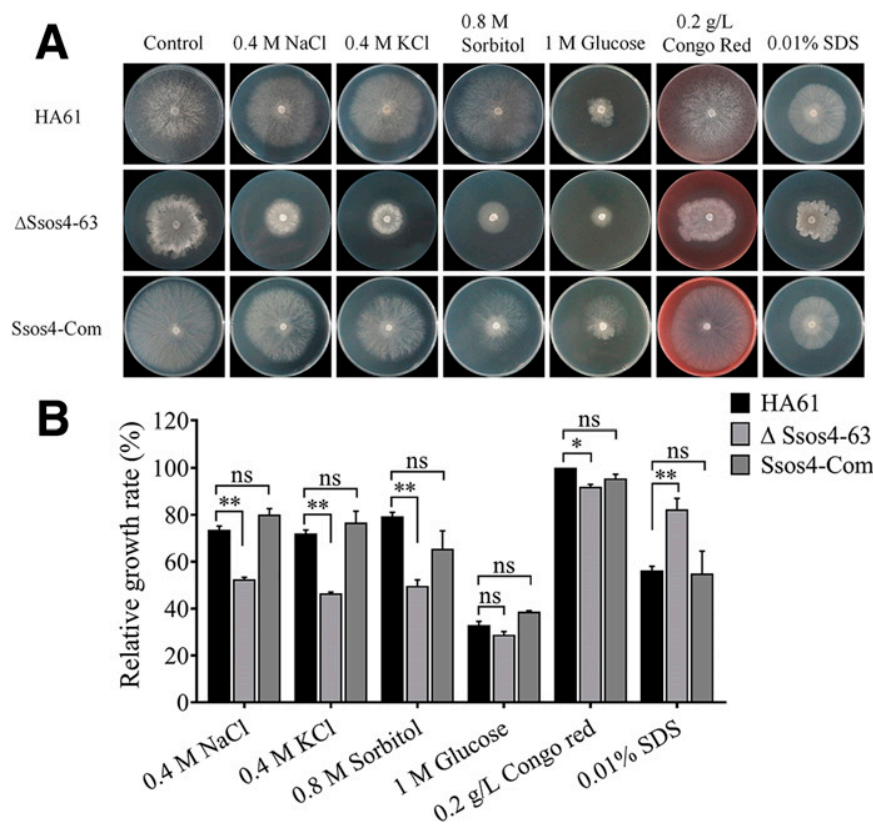

Fig. 5. Responses to cellular stresses. A, Sensitivity of the wild-type progenitor HA61, the deletion mutant $\Delta S \operatorname{sos} 4-63$, and the complementary mutant to osmotic, cell membrane, and cell wall stresses on potato dextrose agar (PDA) plates. B, Relative growth of mutants on PDA plates supplied with stress compounds. SDS $=$ sodium dodecyl sulfate. Asterisks $*$ and $* *$ denote significant differences at $P<0.05$ and 0.01 , respectively, and ns denotes no significant difference at $P<0.05$.

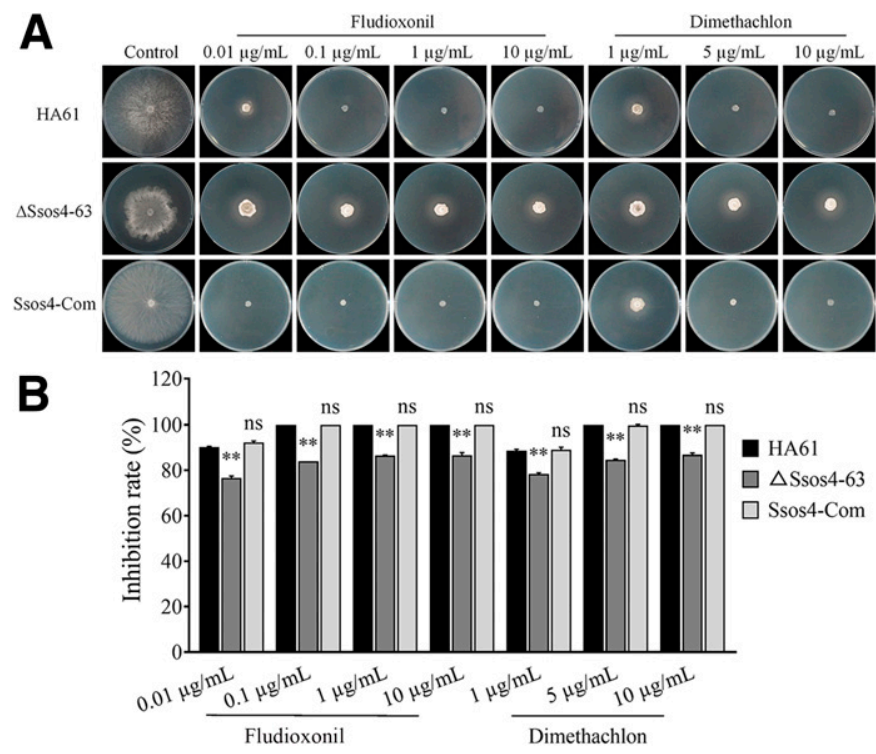

Fig. 6. Sensitivity to fludioxonil and dimethachlon. A, Sensitivity of the wildtype strain HA61, the deletion mutant $\Delta$ Ssos4-63, and the revertant Ssos4-Com on potato dextrose agar (PDA) plates with added fungicides. B, Inhibition rates on PDA plates with fludioxonil and dimethachlon. Asterisks (**) denote significant difference at $P<0.01$ and ns denotes no significant difference at $P<0.05$ deletion on the formation of sclerotia and infection cushion, the expression of key genes related to sclerotia formation and infection cushion (Zhu et al. 2019) was assayed and the results suggested that the deletion of Ssos 4 decreased the expression levels of these genes (Supplementary Figs. S1 and S2). Thus, the survival fitness was widely impaired by the deletion of Ssos4.

The HOG pathway is an important signal transmitting pathway in cells and is involved in hyperosmotic adaptation (de Nadal et al. 2011). In $N$. crassa, the deletion mutants of $N \cos -1, N \cos -2, N \cos -4$, and $N \cos -5$ showed hypersensitivity to osmotic stress (Fujimura et al. 2003). Deletion of Bcos4 in B. cinerea and Fgssk2 in $F$. graminearum both caused increased sensitivity to hyperosmotic stress (Yang et al. 2012; Zheng et al. 2012); similar results were also observed in the deletion mutant $\Delta$ Ssos4-63 (Fig. 5). We speculated that the osmosensitivity was related to the function of Ssk $2 p$ which had been reported for facilitating actin cytoskeleton reassembly after osmotic stress (Yuzyuk et al. 2002). Additionally, the deletion mutant $\Delta$ Ssos4-63 showed more sensitivity to cell wall stresses, which was consistent with the increased sensitivity of the deletion

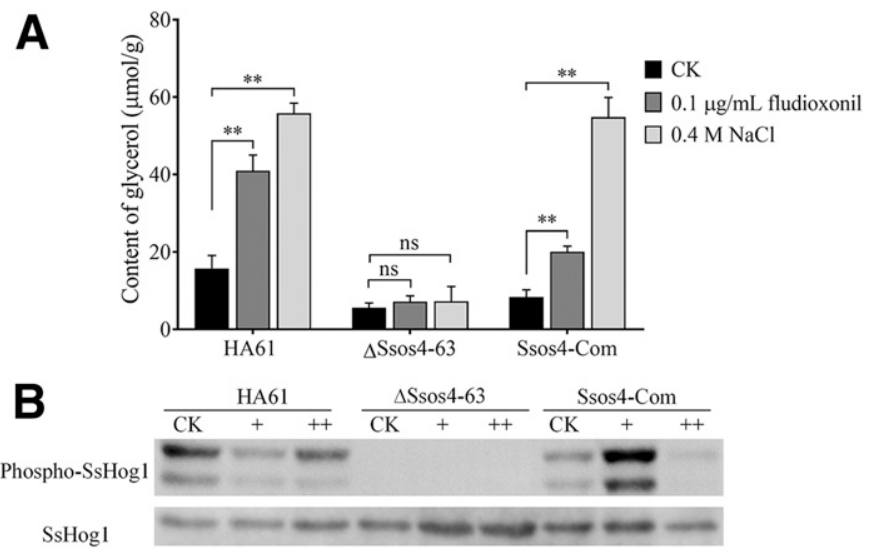

Fig. 7. Glycerol content and the phosphorylation of SsHog1. A, Glycerol content in mycelia treated with fludioxonil at $0.1 \mu \mathrm{g} / \mathrm{ml}$ and $0.4 \mathrm{M} \mathrm{NaCl}$ for 1 h. CK indicates the control treatment. Asterisks $(* *)$ denote significant difference at $P<0.01$. B, Detection of SsHog1 and phosphorylated SsHog1. CK indicates the control treatment, + denotes the treatment of fludioxonil at 0.1 $\mu \mathrm{g} / \mathrm{ml}$ for $1 \mathrm{~h}$, and ++ denotes the treatment of $0.4 \mathrm{M} \mathrm{NaCl}$ for $1 \mathrm{~h}$.

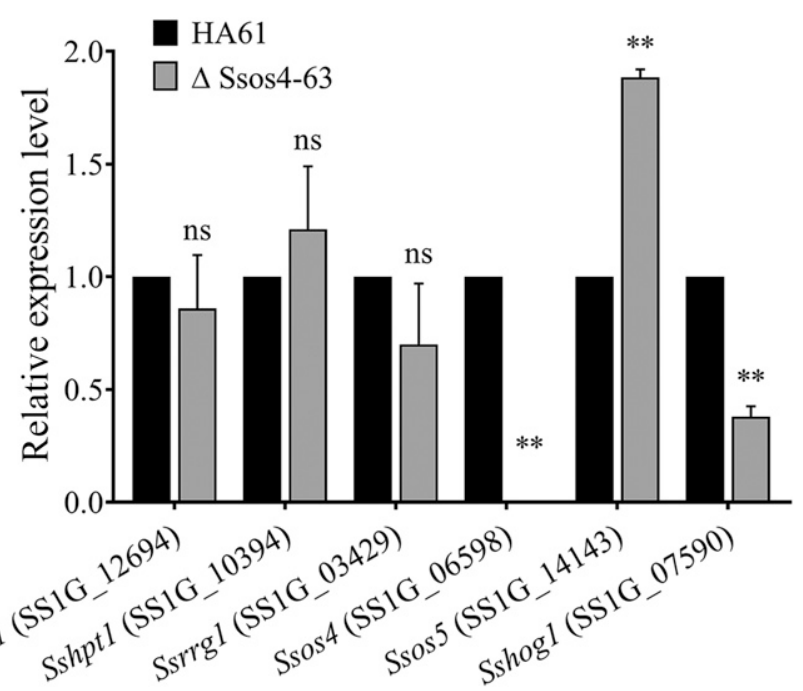

Fig. 8. Relative expression levels of genes in the high osmolarity glycerol pathway. Asterisks (**) denote significant difference at $P<0.01$ and ns denotes no significant difference at $P<0.05$. 
mutant of Fgssk2 to cell wall stresses. The disruption of Fgssk2 showed increased sensitivity to cytoplasm membrane stress (Zheng et al. 2012) but we recorded a contrary result in this study (Fig. 5). This discrepancy might be due to the different crosslinks of MAPK cascades in the various fungi. The alteration of osmotic response in the deletion mutant $\Delta$ Ssos4-63 suggested that Ssos4, as activator of the Hog1 MAPK cascade, played crucial roles in responses to osmotic stresses, cell wall stresses, and cytoplasm membrane stress.

In addition to regulating the responses to osmotic and cell wall stresses, the fungal osmotic signal transduction pathway was considered to be the target of phenylpyrrole and dicarboximide fungicides because the fungal hybrid HKs were involved with the phenylpyrrole resistance (Kilani and Fillinger 2016; Kojima et al. 2004). It has been verified that the deletion of HKs resulted in osmosensitivity and fungicide resistance in Magnaporthe grisea (Motoyama et al. 2005), S. sclerotiorum (Duan et al. 2013b), S. homoeocarpa (Sang et al. 2017), B. cinerea (Viaud et al. 2006), F. oxysporum (Rispail and Pietro 2010), and N. crassa (Fujimura et al. 2000). In spite of HKs, the deficiencies of other genes in the HOG pathway were also reported to be involved in the sensitivity to phenylpyrrole and dicarboximide fungicides. In $N$. crassa, disruption of any one of $N \cos -4, N \cos -5$, and $N \cos$ - 2 caused resistance to fludioxonil and iprodione (Fujimura et al. 2000, 2003). Disruption of the hog 1 gene increased resistance to fludioxonil and iprodione in Penicillium digitatum (Wang et al. 2014) and Verticillium dahliae (Wang et al. 2016). In contrast, increased sensitivity to fludioxonil and iprodione was observed by the deletion of $B \cos 4$ in $B$. cinerea (Yang et al. 2012). In our study, deletion of Ssos 4 significantly increased the $\mathrm{EC}_{50}$ value of fludioxonil, reduced that of dimethachlon, and resulted in survival of the deletion mutant when treated with much higher concentrations of both fungicides (Fig. 6). The decreased sensitivity of the deletion mutant $\Delta$ Ssos4-63 to fungicides indicated that Ssos 4 is involved in the response of $S$. sclerotiorum to the fungicides fludioxonil and dimethachlon, which is consistent with the disruption of $s s k 2$ in C. neoformans (Bahn et al. 2007).

The accumulation of intracellular glycerol, an important phenotype in adaptation to osmotic stress, was tested. Compared

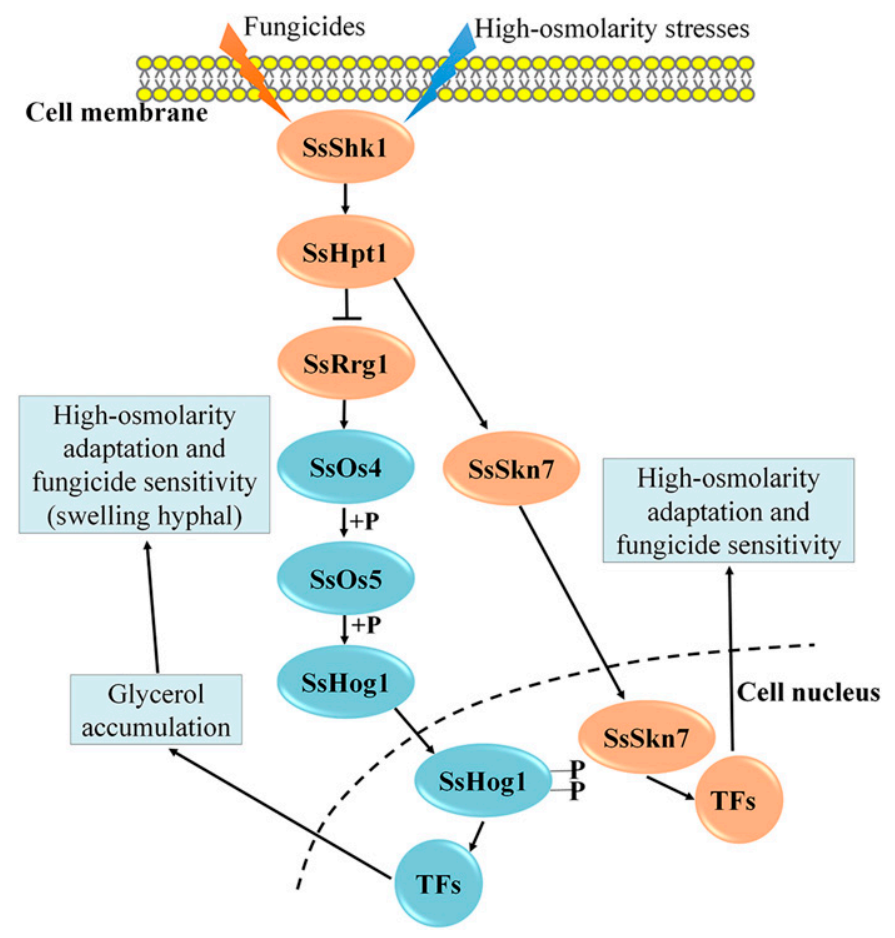

Fig. 9. Proposed mechanism of the high osmolarity glycerol pathway in Sclerotinia sclerotiorum. with the wild-type progenitor HA61, intracellular glycerol accumulation was reduced in the deletion mutant $\Delta$ Ssos4-63 in the treatments control, fludioxonil, and $\mathrm{NaCl}$ (Fig. 7A). Moreover, glycerol accumulation was not significantly induced by fludioxonil and $0.4 \mathrm{M} \mathrm{NaCl}$ in the deletion mutant $\Delta \mathrm{Ssos} 4-63$, which is in line

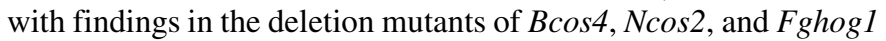
(Yang et al. 2012; Zhang et al. 2002; Zheng et al. 2012). In addition, the transcription levels of SsglpA and Ssgppl, which encode glycerol-3-phosphate dehydrogenase and glycerol-1-phosphatase, respectively, were determined. After exposure to fludioxonil at $0.1 \mu \mathrm{g} / \mathrm{ml}$ and $0.4 \mathrm{M} \mathrm{NaCl}$ for $1 \mathrm{~h}$, the expression level of $S \mathrm{~g} g l p A$ was significantly increased in the wild-type strain HA61 but not in the deletion mutant $\Delta$ Ssos4-63 (Supplementary Fig. S3). No difference in the expression level of Ssgppl was seen between HA61 and $\Delta$ Ssos4-63 (Supplementary Fig. S3). The results are consistent with findings that glycerol export and glycerol-3-phosphate dehydrogenase but not glycerol phosphatase are rate-limiting enzymes for glycerol production (Remize et al. 2001). Overexpression of FPS1, which encodes a glycerol channel protein, enhances glycerol production in Saccharomyces cerevisiae (Luyten et al. 1995). In our study, increased expression of Ssfpsl was also discovered in the wild-type stain HA61 but not in the deletion mutant $\Delta \mathrm{Ssos} 4-63$ after exposure to $0.4 \mathrm{M} \mathrm{NaCl}$ and fludioxonil at $0.1 \mu \mathrm{g} / \mathrm{ml}$ for $1 \mathrm{~h}$ (Supplementary Fig. S3). The phosphorylation of Hog1 is important for the regulation of glycerol accumulation (Irmler et al. 2006; Noguchi et al. 2007). Both osmotic stress and fungicide treatment stimulate the phosphorylation of Hog 1 to regulate the expression of transcription factors in adaptation to the stresses (Irmler et al. 2006). In this study, phosphorylation of SsHog1 was cancelled in the deletion mutant $\Delta$ Ssos4-63 (Fig. 7B). This is equivalent to the $N \cos 4$ mutant in $N$. crassa and the Bcos 4 mutant in $B$. cinerea (Noguchi et al. 2007; Yang et al. 2012). In S. cerevisiae, the control of the Fpsl channel is independent of Hogl under osmotic stress (Luyten et al. 1995) but the connection between increased Ssfps 1 expression and cancelled SsHog1 phosphorylation was uncovered in this study, which is worth exploring. Decreased expression of Sshogl was also observed by the deletion of Ssos 4 as well as the disruption of Bcos4 in B. cinerea (Yang et al. 2012). Considering that the phosphorylation of Hog1 was entirely cancelled by the deletion of $S \operatorname{sos} 4$ and the deletion mutant $\Delta$ Ssos4-63 survived under hyperosmotic stresses and fungicide stresses, we supposed that the regulation of gene expression by $\mathrm{Hog} 1 \mathrm{p}$ was not absolutely necessary for cell survival under certain conditions.

Due to its multinuclear phenomenon, it was difficult to genetically manipulate S. sclerotiorum. In this study, we illustrated the function of Ssos 4, an important kinase in the Hog1 MAPK cascade, and the results showed that Ssos 4 was involved in the regulation of mycelial growth and differentiation, sclerotia formation, virulence, hyperosmotic adaptation, and responses to phenylpyrrole and dicarboximide fungicides. In addition, $\mathrm{Ss}_{\mathrm{O}} \mathrm{S} 4$ was also vital for the phosphorylation of SsHog1 in response to hyperosmotic stress and fungicides. There are two functionally redundant branches upstream of the HOG pathway in yeast but only one in some filamentous fungi (Furukawa et al. 2005). In this study, we confirmed that the Pbs2-Hog1 cascade was only activated by $\mathrm{SsO} 4$ through the Shk1-SsHpt1-SsRrg1 (Sln1Ypd1-Ssk1) branch in S. sclerotiorum. According to the results of our study and the schematic diagram of the HOG pathway in yeast, we developed a proposed mechanism of the HOG pathway in $S$. sclerotiorum (Fig. 9). We supposed that the SsOs4-SsOs5SsHog 1 branch and the SsSkn7 branch are both involved in osmolar adaptation and fludioxonil sensitivity under the control of the HK gene Shk1 in S. sclerotiorum. The SsOs4-SsOs5-SsHog1 cascade regulates glycerol accumulation in response to fludioxonil to cause hyphal swelling but the role of $S s s k n 7$ in acclimatization to a high-osmolarity environment is unclear, though Skn7p is thought to be involved in cell wall integrity under normal and hypoosmotic conditions (Brown et al. 1993, 1994). Given the sensitivity of the Ssos 2 deletion mutant and the resistance of the Ssskn7 deletion 
mutant to fludioxonil (our unpublished data), we considered that the SsSkn7 branch is mainly involved in the response to fludioxonil in $S$. sclerotiorum and should be investigated further.

Although we illustrated that Ssos 4 gene played a node role in the regulatory system of the HOG pathway in S. sclerotiorum, the research on substrates in the HOG pathway is far from complete. Identification and characterization of a two-component system and the Hog1 MAPK cascade will serve to define the regulatory mechanism under their control as well as the mode of action of phenylpyrrole and dicarboximide fungicides.

\section{LITERATURE CITED}

Arthur, J. S. C., and Ley, S. C. 2013. Mitogen-activated protein kinases in innate immunity. Nat. Rev. Immunol. 13:679-692.

Avenot, H., Simoneau, P., Iacomi-Vasilescu, B., and Bataillé-Simoneau, N. 2005. Characterization of mutations in the two-component histidine kinase gene AbNIK1 from Alternaria brassicicola that confer high dicarboximide and phenylpyrrole resistance. Curr. Genet. 47:234-243.

Bahn, Y. S., Geunes-Boyer, S., and Heitman, J. 2007. Ssk2 mitogen-activated protein kinase kinase kinase governs divergent patterns of the stressactivated Hog1 signaling pathway in Cryptococcus neoformans. Eukaryot. Cell 6:2278-2289.

Boland, G. J., and Hall, R. 1994. Index of plant hosts of Sclerotinia sclerotiorum. Can. J. Plant Pathol. 16:93-108.

Bolton, M. D., Thomma, B. P. H. J., and Nelson, B. D. 2006. Sclerotinia sclerotiorum (Lib.) de Bary: Biology and molecular traits of a cosmopolitan pathogen. Mol. Plant Pathol. 7:1-16.

Brown, J. L., Bussey, H., and Stewart, R. C. 1994. Yeast Skn7p functions in a eukaryotic two-component regulatory pathway. EMBO J. 13:5186-5194.

Brown, J. L., North, S., and Bussey, H. 1993. SKN7, a yeast multicopy suppressor of a mutation affecting cell wall beta-glucan assembly, encodes a product with domains homologous to prokaryotic two-component regulators and to heat shock transcription factors. J. Bacteriol. 175:6908-6915.

Cunha, W. G., Tinoco, M. L. P., Pancoti, H. L., Ribeiro, R. E., and Aragão, F. J. L. 2010. High resistance to Sclerotinia sclerotiorum in transgenic soybean plants transformed to express an oxalate decarboxylase gene. Plant Pathol. 59:654-660.

Dalili, A., Bakhtiari, S., Barari, H., and Aldaghi, M. 2015. Effect of some fungicides against the growth inhibition of Sclerotinia sclerotiorum mycelial compatibility groups. J. Plant Prot. Res. 55:354-361.

de Nadal, E., Alepuz, P. M., and Posas, F. 2002. Dealing with osmostress through MAP kinase activation. EMBO Rep. 3:735-740.

de Nadal, E., Ammerer, G., and Posas, F. 2011. Controlling gene expression in response to stress. Nat. Rev. Genet. 12:833-845.

Di, Y. L., Zhu, Z. Q., Lu, X. M., and Zhu, F. X. 2016. Baseline sensitivity and efficacy of trifloxystrobin against Sclerotinia sclerotiorum. Crop Prot. 87: 31-36.

Duan, Y. B., Ge, C. Y., Liu, S. M., Chen, C. J., and Zhou, M. G. 2013a. Effect of phenylpyrrole fungicide fludioxonil on morphological and physiological characteristics of Sclerotinia sclerotiorum. Pestic. Biochem. Physiol. 106: 61-67.

Duan, Y. B., Ge, C. Y., Liu, S. M., Wang, J. X., and Zhou, M. G. 2013b. A twocomponent histidine kinase Shk1 controls stress response, sclerotial formation and fungicide resistance in Sclerotinia sclerotiorum. Mol. Plant Pathol. 14:708-718.

Fujimura, M., Ochiai, N., Ichiishi, A., Usami, R., Horikoshi, K., and Yamaguchi, I. 2000. Fungicide resistance and osmotic stress sensitivity in os mutants of Neurospora crassa. Pestic. Biochem. Physiol. 67:125-133.

Fujimura, M., Ochiai, N., Oshima, M., Motoyama, T., Ichiishi, A., Usami, R., Horikoshi, K., and Yamaguchi, I. 2003. Putative homologs of SSK22 MAPKK kinase and PBS2 MAPK kinase of Saccharomyces cerevisiae encoded by $o s-4$ and $o s-5$ genes for osmotic sensitivity and fungicide. Biosci. Biotechnol. Biochem. 67:186-191.

Furukawa, K., Hoshi, Y., Maeda, T., Nakajima, T., and Abe, K. 2005. Aspergillus nidulans HOG pathway is activated only by two-component signalling pathway in response to osmotic stress. Mol. Microbiol. 56:1246-1261.

Ge, C. Y., Duan, Y. B., Zhou, M. G., and Chen, C. J. 2013. A protoplast transformation system for gene deletion and complementation in Sclerotinia sclerotiorum. J. Phytopathol. 161:800-806.

Godoy, G., Steadman, J. R., Dickman, M. B., and Dam, R. 1990. Use of mutants to demonstrate the role of oxalic acid in pathogenicity of Sclerotinia sclerotiorum on Phaseolus vulgaris. Physiol. Mol. Plant Pathol. 37: 179-191.

Guimarães, R. L., and Stotz, H. U. 2004. Oxalate production by Sclerotinia sclerotiorum deregulates guard cells during infection. Plant Physiol. 136: 3703-3711.
Irmler, S., Rogniaux, H., Hess, D., and Pillonel, C. 2006. Induction of OS-2 phosphorylation in Neurospora crassa by treatment with phenylpyrrole fungicides and osmotic stress. Pestic. Biochem. Physiol. 84:25-37.

Jacob, S., Foster, A. J., Yemelin, A., and Thines, E. 2014. Histidine kinases mediate differentiation, stress response, and pathogenicity in Magnaporthe oryzae. MicrobiologyOpen 3:668-687.

Jacob, S., Foster, A. J., Yemelin, A., and Thines, E. 2015. High osmolarity glycerol (HOG) signalling in Magnaporthe oryzae: Identification of MoYPD1 and its role in osmoregulation, fungicide action, and pathogenicity. Fungal Biol. 119:580-594.

Jamaux, I., Gelie, B., and Lamarque, C. 1995. Early stages of infection of rapeseed petals and leaves by Sclerotinia sclerotiorum revealed by scanning electron microscopy. Plant Pathol. 44:22-30.

Jiang, L. H., Cao, C. L., Lin, W., Xia, J., Xu, H. H., and Zhang, Y. 2014. Cadmium-induced activation of high osmolarity glycerol pathway through its $\operatorname{Sln} 1$ branch is dependent on the MAP kinase kinase kinase Ssk2, but not its paralog Ssk22, in budding yeast. FEMS Yeast Res. 14:1263-1272.

Johnson, D. A., and Atallah, Z. K. 2014. Disease cycle, development and management of Sclerotinia stem rot of potato. Am. J. Plant Sci. 5: 3717-3726.

Jones, C. A., Greer-Phillips, S. E., and Borkovich, K. A. 2007. The response regulator RRG-1 functions upstream of a mitogen-activated protein kinase pathway impacting asexual development, female fertility, osmotic stress, and fungicide resistance in Neurospora crassa. Mol. Biol. Cell 18: 2123-2136.

Kilani, J., and Fillinger, S. 2016. Phenylpyrroles: 30 Years, two molecules and (nearly) no resistance. Front. Microbiol. 7:e1001479.

Kojima, K., Takano, Y., Yoshimi, A., Tanaka, C., Kikuchi, T., and Okuno, T. 2004. Fungicide activity through activation of a fungal signalling pathway. Mol. Microbiol. 53:1785-1796.

Lew, R. R. 2010. Turgor and net ion flux responses to activation of the osmotic MAP kinase cascade by fludioxonil in the filamentous fungus Neurospora crassa. Fungal Genet. Biol. 47:721-726.

Liu, S. M., Duan, Y. B., Ge, C. Y., Chen, C. J., and Zhou, M. G. 2013. Functional analysis of the $\beta 2$-tubulin gene of Fusarium graminearum and the $\beta$-tubulin gene of Botrytis cinerea by homologous replacement. Pest Manage. Sci. 69:582-588.

Liu, S. M., Fu, L. Y., Hai, F., Jiang, J., Che, Z. P., Tian, Y. E., and Chen, G. Q. 2018. Sensitivity to boscalid in field isolates of Sclerotinia sclerotiorum from rapeseed in Henan Province, China. J. Phytopathol. 166:227-232.

Liu, S. M., Jiang, J., Che, Z. P., Tian, Y. E., and Chen, G. Q. 2019. Baseline sensitivity and control efficacy of fluazinam against Sclerotinia sclerotiorum in Henan Province, China. J. Phytopathol. 167:75-81.

Luyten, K., Albertyn, J., Skibbe, W. F., Prior, B. A., Ramos, J., Thevelein, J. M., and Hohmann, S. 1995. Fps1, a yeast member of the MIP family of channel proteins, is a facilitator for glycerol uptake and efflux and is inactive under osmotic stress. EMBO J. 14:1360-1371.

Ma, H. X., Feng, X. J., Chen, Y., Chen, C. J., and Zhou, M. G. 2009. Occurrence and characterization of dimethachlon insensitivity in Sclerotinia sclerotiorum in Jiangsu province in China. Plant Dis. 93:36-42.

Maeda, T., Wurgler-Murphy, S. M., and Saito, H. 1994. A two-component system that regulates an osmosensing MAP kinase cascade in yeast. Nature 369:242-245.

Meng, X., and Zhang, S. 2013. MAPK cascades in plant disease resistance signaling. Annu. Rev. Phytopathol. 51:245-266.

Motoyama, T., Kadokura, K., Ohira, T., Ichiishi, A., Fujimura, M., Yamaguchi, I., and Kudo, T. 2005. A two-component histidine kinase of the rice blast fungus is involved in osmotic stress response and fungicide action. Fungal Genet. Biol. 42:200-212.

Mullins, E. D., Chen, X., Romaine, P., Raina, R., Geiser, D. M., and Kang, S. 2001. Agrobacterium-mediated transformation of Fusarium oxysporum: An efficient tool for insertional mutagenesis and gene transfer. Phytopathology 91:173-180.

Noguchi, R., Banno, S., Ichikawa, R., Fukumori, F., Ichiishi, A., Kimura, M., Yamaguchi, I., and Fujimura, M. 2007. Identification of OS-2 MAP kinasedependent genes induced in response to osmotic stress, antifungal agent fludioxonil, and heat shock in Neurospora crassa. Fungal Genet. Biol. 44: 208-218.

Ochiai, N., Tokai, T., Nishiuchi, T., Takahashi-Ando, N., Fujimura, M., and Kimura, M. 2007. Involvement of the osmosensor histidine kinase and osmotic stress-activated protein kinases in the regulation of secondary metabolism in Fusarium graminearum. Biochem. Biophys. Res. Commun. 363:639-644.

Pan, Y. L., Wang, Z. Y., and Wu, H. Z. 1997. Resistance and genetic stability of Sclerotinia sclerotiorum to carbendazim. Jiangsu J. Agric. Sci. 13:32-35.

Posas, F., Wurgler-Murphy, S. M., Maeda, T., Witten, E. A., and Saito, H. 1996. Yeast HOG1 MAP kinase cascade is regulated by a multistep phosphorelay mechanism in the SLN1-YPD1-SSK1 "two-component" osmosensor. Cell 86:865-875. 
Raman, M., Chen, W., and Cobb, M. H. 2007. Differential regulation and properties of MAPKs. Oncogene 26:3100-3112.

Remize, F., Barnavon, L., and Dequin, S. 2001. Glycerol export and glycerol-3phosphate dehydrogenase, but not glycerol phosphatase, are rate limiting for glycerol production in Saccharomyces cerevisiae. Metab. Eng. 3:301-312.

Rispail, N., and Pietro, A. D. 2010. The two-component histidine kinase Fhk1 controls stress adaptation and virulence of Fusarium oxysporum. Mol. Plant Pathol. 11:395-407.

Sang, H., Popko, J. T., Chang, T., and Jung, G. 2017. Molecular mechanisms involved in qualitative and quantitative resistance to the dicarboximide fungicide iprodione in Sclerotinia homoeocarpa field isolates. Phytopathology 107:198-207.

Shor, E., and Chauhan, N. 2015. A case for two-component signaling systems as antifungal drug targets. PLoS Pathog. 11:e1004632.

Viaud, M., Fillinger, S., Liu, W., Polepalli, J. S., Pêcheur, L. P., Kunduru, A. R., Leroux, R., and Legendre, L. 2006. A class III histidine kinase acts as a novel virulence factor in Botrytis cinerea. Mol. Plant-Microbe Interact. 19:1042-1050

Wang, J. X., Ma, H. X., Chen, Y., Zhu, X. F., Yu, W. Y., Tang, Z. H., Chen, C. J., and Zhou, M. G. 2009. Sensitivity of Sclerotinia sclerotiorum from oilseed crops to boscalid in Jiangsu Province of China. Crop Prot. 28:882-886.

Wang, M., Chen, C., Zhu, C., Sun, X., Ruan, R., and Li, H. 2014. Os2 MAP kinase-mediated osmostress tolerance in Penicillium digitatum is associated with its positive regulation on glycerol synthesis and negative regulation on ergosterol synthesis. Microbiol. Res. 169:511-521.

Wang, Y., Tian, L., Xiong, D., Klosterman, S. J., Xiao, S., and Tian, C. 2016. The mitogen-activated protein kinase gene, VdHogl, regulates osmotic stress response, microsclerotia formation and virulence in Verticillium dahlia. Fungal Genet. Biol. 88:13-23.

Williams, B., Kabbage, M., Kim, H. J., Britt, R., and Dickman, M. B. 2011. Tipping the balance: Sclerotinia sclerotiorum secreted oxalic acid suppresses host defenses by manipulating the host redox environment. PLoS Pathog. 7:e1002107.

Wojda, I., Alonso-Monge, R., Bebelman, J. P., Mager, W. H., and Siderius, M. 2003. Response to high osmotic conditions and elevated temperature in
Saccharomyces cerevisiae is controlled by intracellular glycerol and involves coordinate activity of MAP kinase pathways. Microbiology 149: 1193-1204.

Wu, Y., Xu, L., Liu, J., Yin, Z., Gao, X., Feng, H., and Huang, L. 2017. A mitogen-activated protein kinase gene $(\mathrm{VmPmk} 1)$ regulates virulence and cell wall degrading enzyme expression in Valsa mali. Microb. Pathog. 111: 298-306.

Yang, Q. Q., Yan, L. Y., Gu, Q., and Ma, Z. H. 2012. The mitogen-activated protein kinase kinase kinase $\mathrm{BcOs} 4$ is required for vegetative differentiation and pathogenicity in Botrytis cinerea. Appl. Microbiol. Biotechnol. 96: 481-492.

Yuzyuk, T., Foehr, M., and Amberg, D. C. 2002. The MEK kinase Ssk2p promotes actin cytoskeleton recovery after osmotic stress. Mol. Biol. Cell $13: 2869-2880$

Zhang, S. Q., and Klessig, D. F. 2001. MAPK cascades in plant defense signaling. Trends Plant Sci. 6:520-527.

Zhang, Y., Lamm, R., Pillonel, C., Stephen, L., and Xu, J. R. 2002. Osmoregulation and fungicide resistance: The Neurospora crassa os-2 gene encodes a HOG1 mitogen-activated protein kinase homologue. Appl. Environ. Microbiol. 68:532-538.

Zheng, D. W., Zhang, S. J., Zhou, X. Y., Wang, C. F., Xiang, P., Zheng, Q., and $\mathrm{Xu}$, J. R. 2012. The FgHOG1 pathway regulates hyphal growth, stress responses, and plant infection in Fusarium graminearum. PLoS One 7: e49495.

Zhi, H., Tang, L., Xia, Y. J., and Zhang, J. H. 2013. Ssk1p-independent activation of Ssk2p plays an important role in the osmotic stress response in Saccharomyces cerevisiae: Alternative activation of Ssk2p in osmotic stress. PLoS One 8:e54867.

Zhu, G. L., Yu, G., Zhang, X. H., Liu, J. L., Zhang, Y. H., and Pan, H. Y. 2019. The formaldehyde dehydrogenase SsFdh1 is regulated by and functionally cooperates with the GATA transcription factor SsNsd1 in Sclerotinia Sclerotiorum. mSystems 4:e00397-19.

Zhu, Z. Q., Zhou, F., Li, J. L., Zhu, F. X., and Ma, H. J. 2016. Carbendazim resistance in field isolates of Sclerotinia sclerotiorum in China and its management. Crop Prot. 81:115-121. 